\title{
Accuracy of KinectOne to quantify kinematics of the upper body
}

\section{Authors}

Roman P. Kuster ${ }^{a}$, Bernd Heinlein ${ }^{a}$, Christoph M. Bauer ${ }^{b}$, Eveline S. Graf ${ }^{b}$

\section{Affiliations}

a Zurich University of Applied Sciences

School of Engineering,

Institute of Mechanical Systems

Biomechanical Engineering

Technikumstrasse 9

8401 Winterthur, Switzerland

${ }^{\mathrm{b}}$ Zurich University of Applied Sciences

School of Health Professions,

Institute of Physiotherapy

Technikumstrasse 71

8401 Winterthur, Switzerland

Corresponding author: Eveline S. Graf: eveline.graf@zhaw.ch

Word count (Introduction through Conclusion): 2994

\section{Acknowledgments}

The authors would like to acknowledge the assistance of Mariella Oswald for data collection, Andre Meichtry for statistical consultation, and Christian Schärli for providing software.

There was no study sponsor with influence on this study. 


\begin{abstract}
Motion analysis systems deliver quantitative information, e.g. on the progress of rehabilitation programs aimed at improving range of motion. Markerless systems are of interest for clinical application because they are low-cost and easy to use. The first generation of the Kinect ${ }^{\mathrm{TM}}$ sensor showed promising results in validity assessment compared to an established markerbased system. However, no literature is available on the validity of the new 'Kinect ${ }^{\mathrm{TM}}$ for Xbox one' (KinectOne) in tracking upper body motion. Consequently, this study was conducted to analyze the accuracy and reliability of the KinectOne in tracking upper body motion.
\end{abstract}

Twenty subjects performed shoulder abduction in frontal and scapula plane, flexion, external rotation and horizontal flexion in two conditions (sitting and standing). Arm and trunk motion were analyzed using the KinectOne and compared to a marker-based system. Comparisons were made using Bland Altman statistics and Coefficient of Multiple Correlation.

On average, differences between systems of $3.9 \pm 4.0^{\circ}$ and $0.1 \pm 3.8^{\circ}$ were found for arm and trunk motion, respectively. Correlation was higher for the arm than for the trunk motion.

Based on the observed bias, the accuracy of the KinectOne was found to be adequate to measure arm motion in a clinical setting. Although trunk motion showed a very low absolute bias between the two systems, the KinectOne was not able to track small changes over time. Before the KinectOne can find clinical application, further research is required analyzing whether validity can be improved using a customized tracking algorithm or other sensor placement, and to analyze test-retest reliability.

Keywords: Kinect ${ }^{\mathrm{TM}}$ for Xbox one; kinematics; upper extremity; validity

\title{
Introduction
}


The quantitative description of human motion finds application in research and in clinical settings. A common approach is with marker-based systems (MBS), where markers are placed on the skin. Such systems are used widely in research laboratories and are highly accurate [1]. However, their use has disadvantages: data collection and processing are timeconsuming, require highly trained personnel, and are restricted to the laboratory setting. Markerless systems have evolved alongside the technical advancement of cameras and sensors. The Kinect ${ }^{\mathrm{TM}}$ from Microsoft, which was developed to control video games through body movements, has become of interest to the research community. The Kinect ${ }^{\mathrm{TM}}$ is able to track three-dimensional motion by combining information from a color camera and a depthsensing infrared camera. It is of particular interest for clinical settings, since it is relatively low-cost, does not require time-consuming setup, can be used in various spaces and is easy to use.

In order for the Kinect ${ }^{\mathrm{TM}}$ to be used in clinical settings from a biomechanical perspective, the system needs to have sufficient validity to measure kinematic changes. This would allow, for example, determining the reduced shoulder range of motion (ROM) of a frozen shoulder patient and the monitoring of their progress during physiotherapy on a monthly basis. To achieve this, the system needs a measurement error of ROM of less than $7.7^{\circ}$ (flexion), $6^{\circ}$ (abduction) and $3.7^{\circ}$ (rotation) [2].

Different studies have examined the accuracy of the Kinect for tracking the human body. For shoulder abduction in the frontal plane, a good correlation of ROM between the Kinect and a MBS was found; while for elbow flexion in the sagittal plane, a decreased correlation was obtained [3]. Accordingly, a larger bias for shoulder flexion than abduction was reported [4]. This indicates a dependability of the validity of the Kinect on the plane of motion. Generally, larger differences in kinematic measures were found for lower extremities compared to upper extremities [3,5-7]. Clark et al. found a bias proportional to the measured value of Kinect compared to a MBS for the pelvis and sternum, but not for the hand [8], while others noticed a poorer correlation for the trunk than the shoulder angle for the Kinect compared to a MBS 
[4]. This shows a difference in validity between the core of the body and the extremity for the Kinect. Most studies examined accuracy in the standing position $[3,4,8]$

Most previous studies were executed with the first generation Kinect (KinectV1) [3-8]. In 2014, the new Kinect ${ }^{\mathrm{TM}}$ for Xbox one (KinectOne) was released by Microsoft. This system is based on higher quality sensor technology (1920x1080 instead of $640 \times 480$ resolution for the

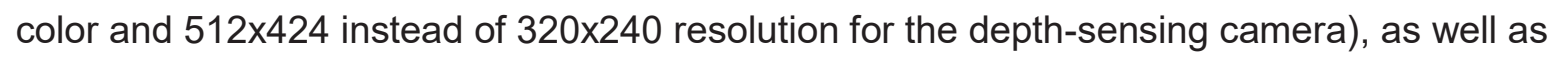
an enlarged field of view compared to KinectV1. Additionally, according to the manufacturer's specification, the algorithm for motion detection has been improved.

It can be speculated that the technological improvements result in higher accuracy in body tracking and, consequently, a higher validity of KinectOne to track movements. A study has found that, generally, KinectOne has excellent concurrent validity for spatiotemporal measurements and anterior-posterior measures during dynamic and static balance tests, but consistently poor to modest validity for kinematic parameters of the lower body and mediallateral measures during balance tests $[9,10]$. Therefore, the aim of this study was to determine the concurrent validity and intra-session reliability of the KinectOne compared to a MBS for measuring segment angles of the trunk and upper extremities during functional movements.

\section{Methods}

Twenty subjects participated (age: mean \pm SD: $33 \pm 9$ years; height: $173.7 \pm 8.4 \mathrm{~cm}$; weight $65.9 \pm 10.6 \mathrm{~kg} ; 10$ female) and signed informed written consent. The study was approved by the local ethics committee. The subjects wore tight-fitting shorts (women with bra). Before data collection, each subject was equipped with 39 reflective markers, according to the plugin-gait full body model [11]. Data were simultaneously collected using a 6-camera Vicon System (200 Hz, VICON, UK) and the KinectOne (30 Hz, Microsoft, USA). The KinectOne was placed $2.5 \mathrm{~m}$ in front of the subject at $1.2 \mathrm{~m}$ above the ground. To synchronize the two 
systems, subjects performed a shoulder abduction movement at the start and the end of each recording.

Five exercises were performed: shoulder abduction (ABD); shoulder abduction in scapular plane (SCAP); shoulder flexion (FLEX); shoulder external rotation with a $90^{\circ}$ flexed elbow (ER); and shoulder horizontal flexion from a starting position in $90^{\circ}$ abduction (HFL) (Figure 1). ABD, SCAP, and FLEX were categorized as inclination, ER and HFL as rotation exercises. Each exercise was performed in sitting and standing, resulting in 10 trials per subject. Each trial consisted of 5 repetitions. Performance of the exercises with the left or the right side was randomly allocated.

The coordinates of the joint centers recorded by KinectOne were stored in a csv-file using custom software based on Kinect for Windows Software Development Kit 2.0. To achieve the same output for MBS data, trajectories of joint centers were calculated using the plug-in-gait model of Vicon Nexus (Version 1.8.5). KinectOne and MBS data were low-pass filtered (Butterworth 2nd order, cut-off frequency 2Hz) using Matlab (Version 2014a, The MathWorks Inc., USA). MBS data were down-sampled to the recording frequency of KinectOne. Data from both systems were synchronized using a cross-correlation based phase shift technique of initial and end abduction [12] and cut into individual repetitions to analyze repetitions two to four.

The body segments were defined by two joint centers (Figure 1). Angular trajectories relative to the initial position were calculated for each segment in the following planes: frontal plane for trunk during $A B D$, SCAP, FLEX and arm during ABD; horizontal plane for trunk and arm during ER and HFL; sagittal plane for arm during FLEX; and scapula plane for arm during SCAP (definitions of planes in the supplementary material).

The Coefficient of Multiple Correlation (CMC) was calculated to exclude repetitions with asynchronous angular trajectories (defined by a complex number of $\mathrm{CMC}$, indicating that the variability of the waveforms around their mean waveform is considerably larger than the 
variance about their grand mean) $[13,14]$. If two or more repetitions resulted in complex numbers, the data of this subject were excluded from the analysis of this exercise $(8 / 2$ data set for trunk/arm were excluded). Additionally, MBS data of one trial could not be analyzed. ROM was calculated as the difference between initial and maximum segment orientation for every repetition and then averaged. To compare agreement between measurement systems, mean bias and limits of agreement (LoA) with 95\% confidence intervals $(\mathrm{Cl})$ were calculated [15]. To analyze intra-session reliability, repeatability coefficient $(R C$, equal to smallest real difference (SRD)) was calculated and compared to LoA to check whether the lack of agreement was caused by a lack of reliability $[15,16]$. Additionally, CMC was calculated to analyze temporal similarities, taking into account the repeated measurement setting [13]. Biases were compared to check for effects of the measurement systems regarding segment, exercise and condition. For segmental comparison, data were evaluated after adjusting for ROM (log-transformation recommended by [15]) and expressed relative to ROM. To compare plane of motion, the additional distinction between exercises of inclination and rotation was made. Statistical comparison was done using nonparametric, classical balanced two-way analysis of variance (Friedman). The level of significance was set to 0.05 . Differences between individual segments, exercises and conditions were specified using the post-hoc Wilcoxon test. If differences between systems were found, the absolute bias was analyzed (regardless of bias direction for absolute accuracy comparison).

\section{Results}

On average, between-system differences of $3.9 \pm 4.0^{\circ}$ and $0.1 \pm 3.8^{\circ}$ were found for arm and trunk motion, respectively. For inclination and rotation exercises the direction of bias for the arm was positive (overestimation). Contrary, KinectOne overestimated inclination of the trunk $\left(2.4 \pm 2.8^{\circ}\right)$ but underestimated rotation $\left(-3.3 \pm 2.0^{\circ}\right)$. RC was found to be smaller than the range from lower to upper LoA for both segments in all exercises (Table 1). Results of 
system comparisons are shown in Table 1 (absolute and relative values) and Figure 2 for the seated condition.

Comparing trunk and arm motion revealed a significantly smaller relative bias for the arm $(p<0.01)$ for all exercises. Inclination of the trunk was overestimated $(21.7 \pm 25.1 \%)$ and rotation underestimated $(-29.3 \pm 8.0 \%)$ by KinectOne. Overestimation of the arm motion was significantly smaller (inclination $2.6 \pm 3.2 \%$, rotation $2.9 \pm 1.5 \%$ ). The mean range from lower to upper LoA was smaller for the arm than the trunk (Table 2). Accordingly, temporal agreement was higher and RC was smaller for the arm than for the trunk.

No general difference of the bias was found between inclination and rotation exercises, although CMC was slightly higher for inclination than rotation (Table 2). Additionally, rotation exercises showed a higher range between lower and upper LoA and a slightly increased RC than inclination. The difference was mainly caused by the large range of LoA of the arm rotation $\left(43.0 \pm 12.7^{\circ}\right)$. Comparing inclination exercises, a significantly higher bias was found for the arm motion in FLEX than in SCAP and ABD in both conditions $(p<0.01)$ (Figure 3$)$. For the trunk, statistical difference was found between the two rotation exercises (bias in ER smaller than in HFL, p<0.01) (Figure 3). Other exercises revealed no differences.

Independently of segment and plane of motion, the bias between the two systems was smaller in sitting than in standing (Table 2). Differences were mainly found for inclination (bias sitting vs. standing: $1.5^{\circ}$ vs. $5.0^{\circ}, \mathrm{p}<0.01$ ) (Figure 3 ). Particularly for the trunk, no differences between the systems were found in seated inclination exercises based on 95\% $\mathrm{Cl}$ of bias (Table 1), while mean inclination bias of standing exercises was significantly higher $\left(+4.8 \pm 1.5^{\circ}, p<0.01\right)$. However, temporal agreement did not differ between the two conditions and $\mathrm{RC}$ was in the same range (Table 2). 


\section{Discussion}

To determine the accuracy of KinectOne in tracking human motion we analyzed concurrent validity and intra-session reliability of KinectOne and a MBS. The motion of the arm and the trunk were recorded in different planes of motion while sitting and standing using both systems simultaneously.

Literature reports lower accuracy of KinectV1 in tracking trunk motion compared to motion of the upper extremities $[4,8]$. Our data showed the opposite result for the absolute bias. However, we have to consider that ROM of the arm exceeded $85^{\circ}$ in all exercises, while the trunk motion never exceeded $20^{\circ}$. Although we found that the biases of both segments were independent of ROM, as has been shown in other studies $[3,4]$, means and standard deviations of the differences between the systems were not the same for both segments. Therefore, a relative comparison is most appropriate [15]. Subsequently, the bias of the arm angle was significantly smaller compared to trunk angle, except for the three inclination exercises in sitting. The SRD for the trunk lay in the range between $42 \%$ and $415 \%$ of ROM, while the same range was lower for the arm (7\% to $38 \%)$. For ABD sitting, this means that a measured change in ROM of the arm of $7 \%$ can be attributed to a real change, while changes below may result from inaccuracies of the sensor. For both systems, RC was found to be smaller compared to the range of the LoA (Table 1), indicating that the lack of agreement between the systems is not caused by a lack of repeatability [15].

KinectOne uses a color camera and an infrared camera to record three-dimensional movements. It can be assumed that depending on the plane of movement, different information from the cameras is needed. For example, it is suspected that shoulder flexion (sagittal plane) relies more on the depth sensor and less on the color camera compared to shoulder abduction (frontal plane). Therefore, we investigated movements in all three spatial directions and in combination (SCAP). For the arm, the poorest accuracy was found for FLEX in the sagittal plane and no difference between the scapular and frontal plane was noticed. During FLEX, the hand was occluding the view of the elbow and shoulder to 
KinectOne at shoulder flexion of about $90^{\circ}$. Although we only used start and end positons to calculate ROM, this occlusion seems to significantly influence the accuracy of tracking the shoulder flexion with KinectOne, in both seated and standing conditions. Interestingly, the tracking of the trunk was not affected by the occlusion. It can be speculated that, when there is occlusion in the frontal view, placing the KinectOne out of plane with a diagonal view of the subject [6] could improve segmental tracking.

A similar observation was made for the trunk rotation in the horizontal plane during HFL, when the arm covered the view of KinectOne to the shoulder center at horizontal flexion above approximately $100^{\circ}$. We found a higher bias for HFL than for ER. This difference was slightly more pronounced in sitting than in standing, probably due to the different sensor height relative to body height.

Contrary to previous literature $[3,6]$, KinectOne had no problem in tracking the arm close to the upper body with $90^{\circ}$ flexed elbow, since the bias for ER was very similar to the bias in ABD and SCAP. However, absolute range from lower to upper LoA was highest for the arm motion in ER (sitting and standing) and thus SRD was above $20^{\circ}$.

In rehabilitation, most exercises are performed in sitting, although previous literature has focused on estimating accuracy of the Kinect sensor in standing [3,4]. Therefore, the results of both body positions were compared to each other. In sitting, a significantly smaller trunk bias was found for all inclination exercises compared to standing. The ROM of the trunk was smaller in sitting than in standing; but the difference remained significant when considering the relative bias. Since the placement of the KinectOne was maintained at the same position for all measurements (1.2 m above the floor), KinectOne had a different view of the subject in the seated compared to the standing exercises, due to the altered body height in the seated tasks. This may explain this difference. It is also possible that Microsoft has incorporated a different tracking algorithm to record joint centers of a seated subject compared to a standing one. Since the Microsoft tracking algorithm is unknown, the use of a custom algorithm, as previously proposed [17], could be beneficial to data improvement. Nevertheless, based on 
the current data it is recommended that the KinectOne is placed at the height of the segment of interest and that shoulder measurements be performed in sitting.

Using the same method as the current study but with KinectV1, Galna et al. investigated arm motion in people with Parkinson's disease and healthy controls [4]. For the controls, they found a mean bias of KinectV1 for standing shoulder abduction of $9^{\circ}\left(\mathrm{LoA}:-1^{\circ}\right.$ to $\left.19^{\circ}\right)$ and for shoulder flexion of $10^{\circ}$ (LoA: $5^{\circ}$ to $15^{\circ}$ ). Bias and LoA of the Parkinson's group was in the same range, although with a smaller ROM. In comparison, our study using KinectOne found a bias for arm abduction that was smaller $\left(0.4^{\circ}\right)$ and a fairly similar bias for arm flexion $\left(11.8^{\circ}\right)$ with a higher ROM. Assessing accuracy of KinectV1, Bonnechère et al. found a mean bias relative to the MBS of $0.4^{\circ}$ for arm abduction at a ROM of $110^{\circ}$ [3]. As in our study, they noticed no bias dependency of ROM. Additionally, they observed the same reproducibility of KinectV1 compared to the MBS. Our repeatability coefficients, indicating the minimum change necessary to detect a true change in ROM, were found to be only slightly increased compared to the MBS, and constantly below the LoA. In conclusion, variation in intra-session reliability observed in this study does not explain the differences in agreement of the two systems.

To compare both systems, data processing for the MBS and the KinectOne were done identically using the plug-in gait joint centers for segmental tracking. Although this method is not common in motion analysis, it is the only option that compares the systems rather than the method of data processing. However, the calculation from superficial bony landmarks to profound joint centers remains subject to an unknown error as the plug-in gait model has limitations in tracking of upper body kinematics. However, it is currently the clinical standard for movement analysis [4]. To analyze trunk inclination, the shoulder centers were used. Other trunk marker nodes of the KinectOne (e.g. sternum and pelvis center), as used by Clark et al. [8], cannot be clearly assigned to an anatomical body point and therefore could not be analyzed with the MBS. Using the shoulder centers to analyze trunk motion results in an overestimation, since the shoulder centers move cranially during abduction. 
The two measurement systems were not aligned to each other. Consequently, a subjectdependent definition of the planes of motion based on the position of the shoulder centers was used. The plane deviations between the two systems depend on the accuracy of tracking of the shoulder centers in the anterior-posterior direction. However, the method used to calculate the angles is relatively stable for small deviations [18] and, therefore, appropriate for this study.

To analyze reliability, the intra-individual variance was used to calculate $\mathrm{RC}$ and compare it to the LoA. This approach allowed the analysis of intra-session reliability, but not intersession reliability. In a subsequent study, a study design to analyze inter-session reliability should be chosen. Before KinectOne can find application in the clinical context, the system needs to be improved in terms of ease of data collection, processing, analysis and reporting through the development of appropriate software that is recognized by quality control services in respective health care settings.

\section{Conclusion}

The results of this study revealed that the accuracy of KinectOne in tracking arm motion is sufficient for clinical settings, with the exception of standing shoulder flexion. The recommendation is that the movements be performed seated. Although absolute bias of trunk motion was generally smaller, KinectOne is not able to track small changes in trunk motion due to the high RC/SRD and low CMC. Future research is needed to improve tracking of the trunk, and to establish whether a different placement and orientation of the KinectOne could improve body tracking. 


\section{Conflict of interest statement}

1

2

None of the authors have any financial or personal relationship with other people or organizations that could inappropriately influence their work. 


\section{Figure Captions}

Figure 1: Start (1) and end position (2) for shoulder flexion (FLEX), external rotation (ER), and horizontal flexion (HFL) in sitting. Note that start and end position of FLEX, ABD and SCAP would be the same if a range of motion of $180^{\circ}$ were performed. Vector from S1 to S2 (shoulder centers) defines the trunk, vector from S1 to E (elbow center) defines the arm.

Figure 2: Average angle curves $(n=20)$ for three movements in seated condition: abduction (ABD: $a, b)$; external rotation (ER: c,d); horizontal flexion (HFL: e,f). Solid line is KinectOne (with standard deviations), dashed line is MBS (with standard deviations). a, c, and e are trunk angles; $b, d$, and $f$ are arm angles. (Curves for abduction in scapula plane and flexion look similar to $A B D$ )

Figure 3: Boxplot of absolute bias between the two measurement systems for abduction in frontal (ABD) and scapular plane (SCAP), flexion (FLEX), external rotation (ER) and horizontal flexion (HFL) in sitting and standing for the trunk and the arm $\left({ }^{*}: p \leq .05,{ }^{* *}: p \leq .01\right)$. The crosses with arrows mark the outliers with values above $15^{\circ}$ (trunk) and $25^{\circ}(\mathrm{arm})$, respectively. 


\section{References}

[1] Richards JG. The measurement of human motion: A comparison of commercially available systems. Hum Movement Sci 1999; 18(5): 589-602

[2] Yang JL, Chang CW, Chen SY, Lin JJ. Shoulder kinematic features using arm elevation and rotation tests for classifying patients with frozen shoulder syndrome who respond to physical therapy. Manual Ther 2008; 13(6): 544-51

[3] Bonnechère B, Jansen B, Salvia P, Bouzahouene H, Omelina L, Moiseev F, et al. Validity and reliability of the Kinect within functional assessment activities: comparison with standard stereophotogrammetry. Gait Posture 2014; 39(1): 593-8

[4] Galna B, Barry G, Jackson D, Mhiripiri D, Olivier P, Rochester L. Accuracy of the Microsoft Kinect sensor for measuring movement in people with Parkinson's disease. Gait Posture 2014; 39(4): 1062-1068

[5] Clark RA, Pua YH, Bryant AL, Hunt Ma. Validity of the Microsoft Kinect for providing lateral trunk lean feedback during gait retraining. Gait Posture 2013; 38(4): 1064-6

[6] Pfister A, West AM, Bronner S, Noah JA. Comparative abilities of Microsoft Kinect and Vicon 3D motion capture for gait analysis. J Med Eng Technol 2014; 38(5): 274-280 [7] Xu X, McGorry RW, Chou L-S, Lin J-h, Chang C-c. Accuracy of the Microsoft Kinect ${ }^{\mathrm{TM}}$ for measuring gait parameters during treadmill walking. Gait Posture 2015; 42(2): 145-151 [8] Clark Ra, Pua Y-H, Fortin K, Ritchie C, Webster KE, Denehy L, et al. Validity of the Microsoft Kinect for assessment of postural control. Gait Posture 2012; 36(3): 372-377 [9] Mentiplay BF, Perraton LG, Bower KJ, Pua Y-H, McGaw R, Heywood S, et al. Gait assessment using the Microsoft Xbox One Kinect: Concurrent validity and inter-day reliability of spatiotemporal and kinematic variables. J Biomech. 2015; 48(10): 2166-2170 
[10] Clark RA, Pua Y-H, Oliveira CC, Bower KJ, Thilaraja S, McGaw R, et al. Reliability and concurrent validity of the Microsoft Xbox One Kinect for assessment of standing balance and postural control. Gait Posture 2015, 42(2): 210-213

[11] Davis RB, Ounpuu S, Tyburski D, Gage JR. A gait analysis data collection and reduction technique. Hum Movement Sci 1991; 10(5): 575-87

[12] Li L, Caldwell GE. Coefficient of cross correlation and the time domain correspondence. J Electromyogr Kines 1999; 9(6): 385-389

[13] Ferrari A, Cutti AG, Cappello A. A new formulation of the coefficient of multiple correlation to assess the similarity of waveforms measured synchronously by different motion analysis protocols. Gait Posture 2010; 31(4): 540-542

[14] Kadaba MP, Ramakrishnan HK, Wootten ME, Gainey J, Gorton G, Cochran GV. Repeatability of kinematic, kinetic, and electromyographic data in normal adult gait. J Orthop Res 1989; 7(6): 849-860

[15] Bland JM, Altman DG. Measuring agreement in method comparison studies. Stat Methods Med Res 1999; 8(2): 135-160

[16] Beckerman H, Roebroeck ME, Lankhorst GJ, Becher JG, Bezemer PD, Verbeek aLM. Smallest real difference, a link between reproducibility and responsiveness. Qual Life Res 2001; 10(7): 571-578

[17] Auvinet E, Multon F, Meunier J. New lower-limb gait asymmetry indices based on a depth camera. Sensors 2015; 15(3): 4605-4023

[18] Crawford NR, Yamaguchi GT, Dickman CA. A new technique for determining 3-D joint angles: the tilt/twist method. Clin Biomech 1999; 14(3): 153-165 


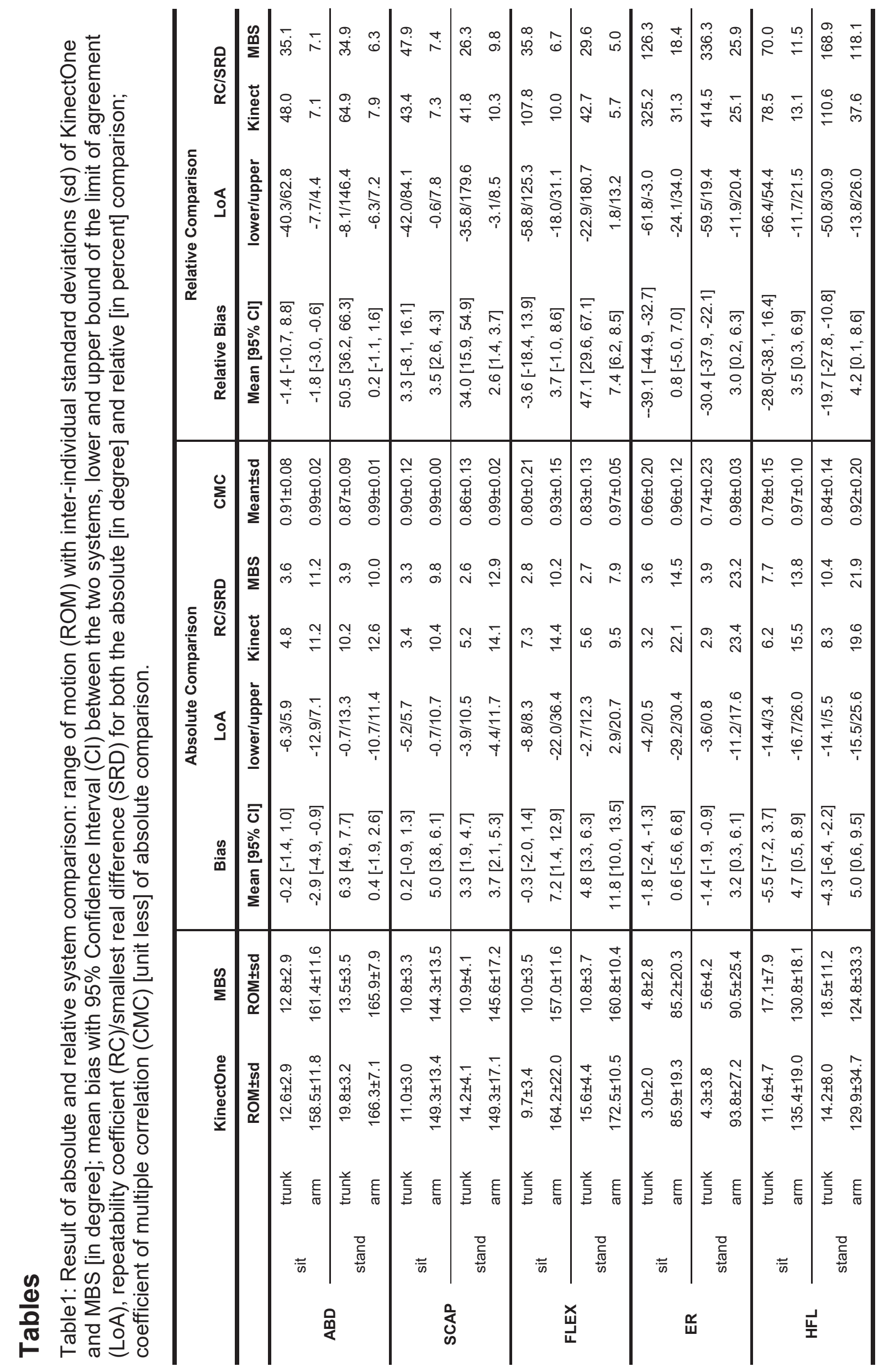


Table 2:Mean results over all trials for the three comparisons made (segment, plane of motion, condition). Indicated are the bias between the two systems, lower and upper bound of the limit of agreement (LoA), the repeatability coefficient (RC)/smallest real difference (SRD), and the coefficient of multiple correlation (CMC) (data of segment comparison in percent of range of motion, other data in degree, CMC unit less).

\begin{tabular}{llcccc}
\hline & Bias & $\begin{array}{c}\text { Range of } \\
\text { LoA }\end{array}$ & $\begin{array}{c}\text { RC/SRD } \\
\text { Kinect One }\end{array}$ & CMC \\
\hline \multirow{2}{*}{ segment } & trunk & $1.3 \pm 32.6 \%$ & $132.7 \pm 54.8 \%$ & $127.8 \pm 131.8 \%$ & $0.82 \pm 0.08$ \\
& arm & $2.7 \pm 2.5 \%$ & $26.9 \pm 18.0 \%$ & $15.5 \pm 11.5 \%$ & $0.97 \pm 0.03$ \\
\hline \multirow{2}{*}{$\begin{array}{l}\text { plane of } \\
\text { motion }\end{array}$} & inclination & $3.3 \pm 4.1^{\circ}$ & $19.1 \pm 12.8^{\circ}$ & $9.1 \pm 3.7^{\circ}$ & $0.92 \pm 0.07$ \\
\hline \multirow{2}{*}{ condition } & rotation & $0.1 \pm 4.0^{\circ}$ & $27.3 \pm 19.5^{\circ}$ & $12.7 \pm 8.5^{\circ}$ & $0.86 \pm 0.12$ \\
& sit & $0.7 \pm 3.9^{\circ}$ & $25.5 \pm 20.3^{\circ}$ & $9.9 \pm 6.1^{\circ}$ & $0.89 \pm 0.11$ \\
& stand & $3.3 \pm 4.4^{\circ}$ & $19.3 \pm 9.9^{\circ}$ & $11.2 \pm 6.5^{\circ}$ & $0.90 \pm 0.08$ \\
\hline
\end{tabular}



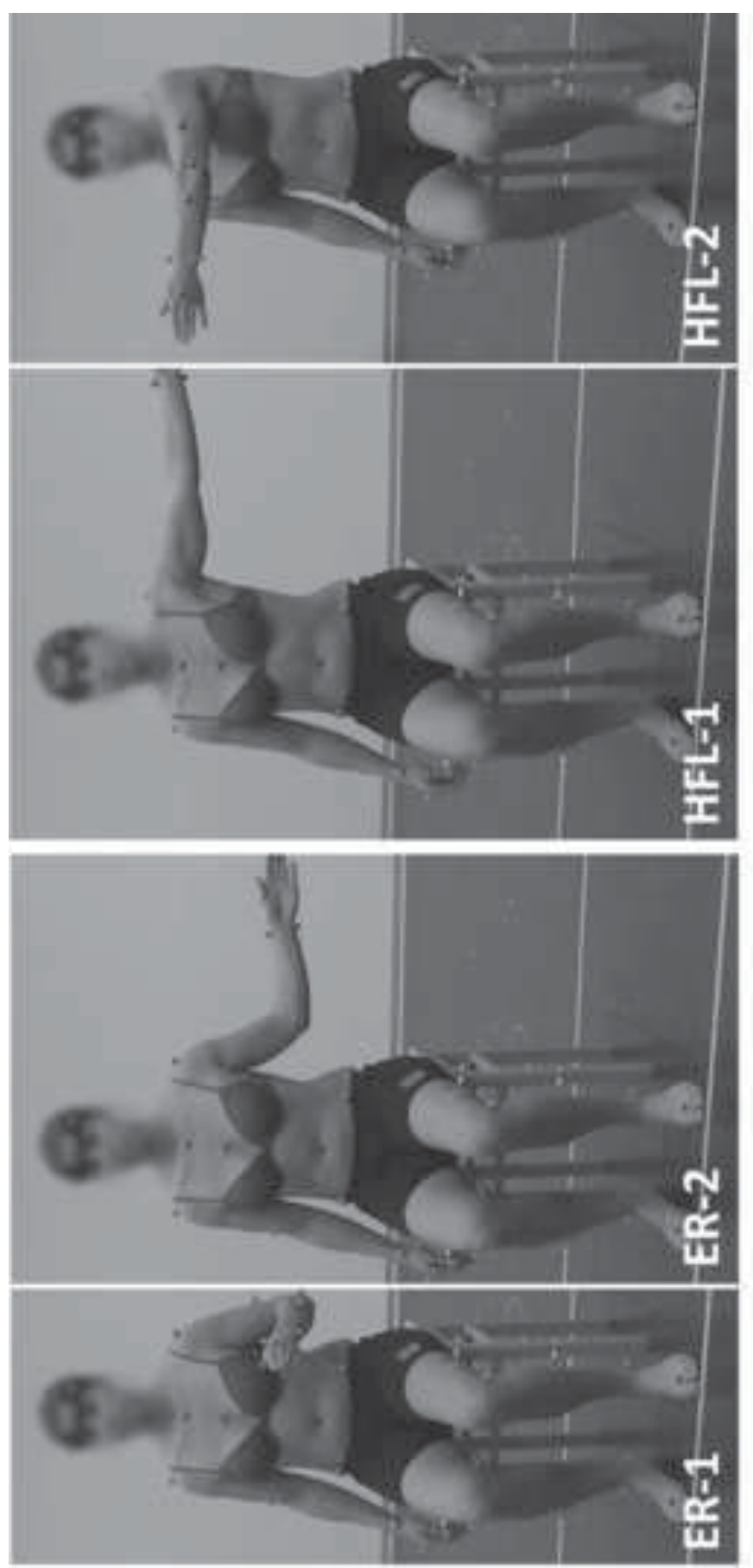

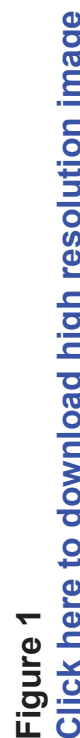
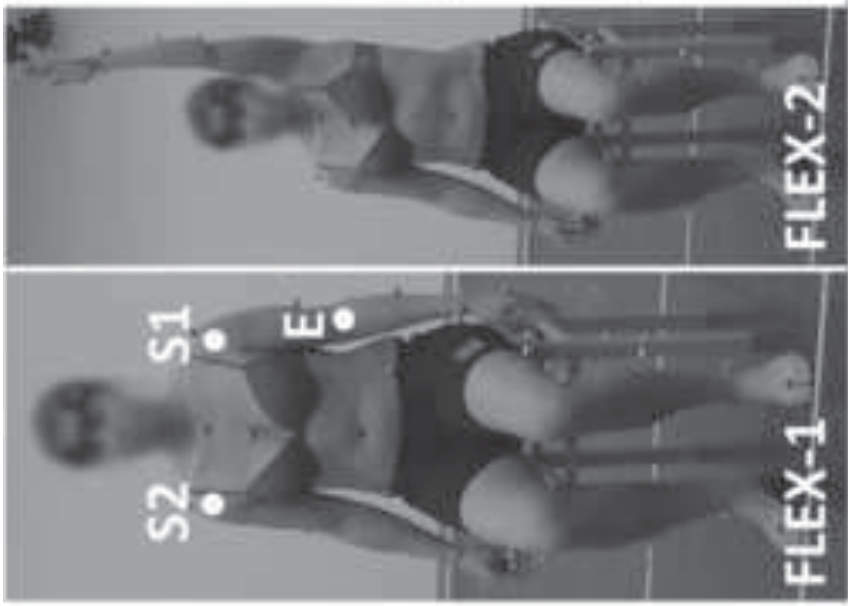

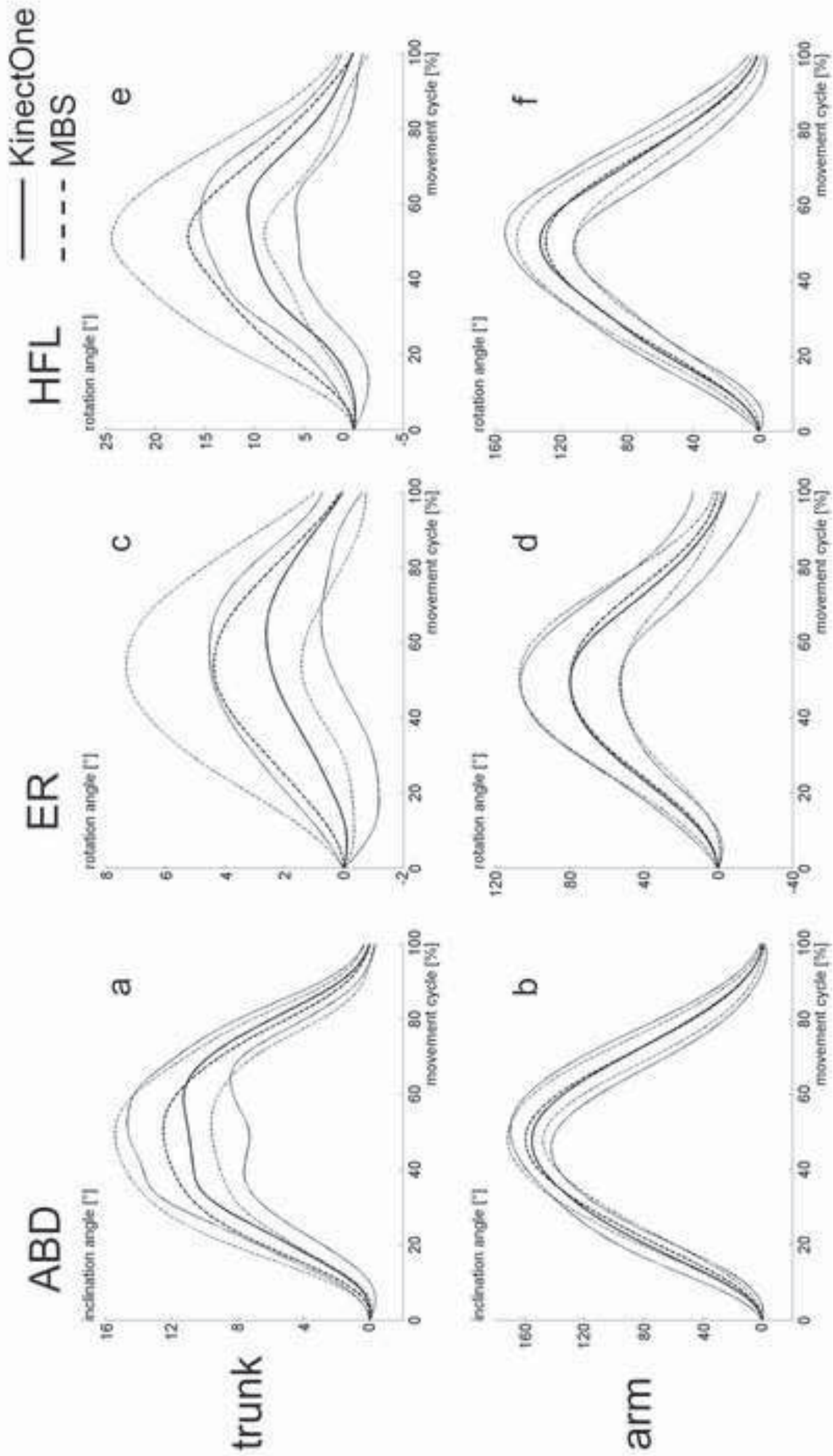

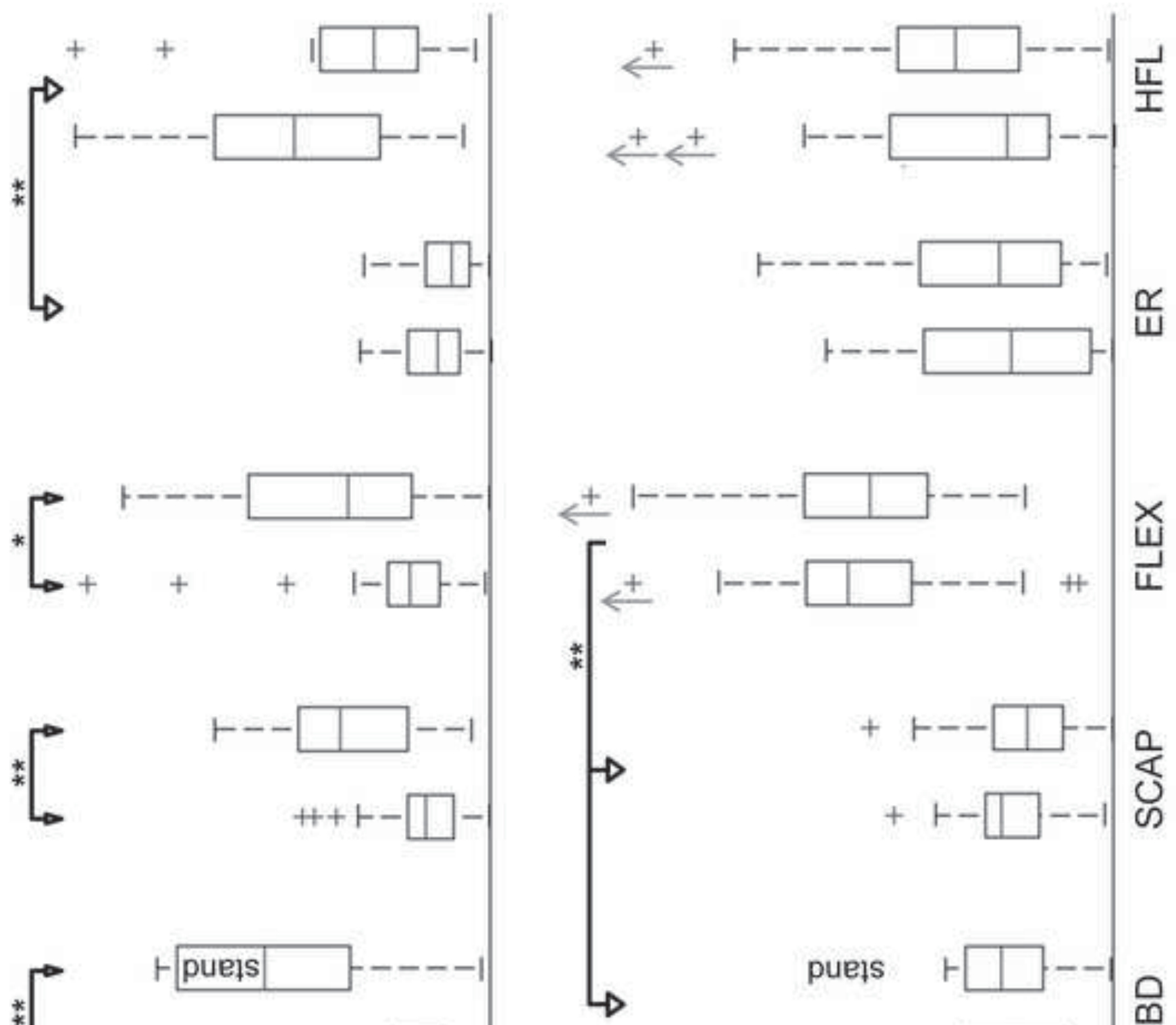

$* \prod_{0}^{\infty}$

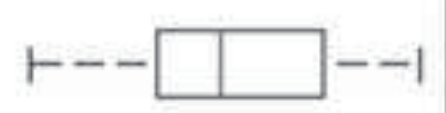

$\frac{\alpha}{u}$

을
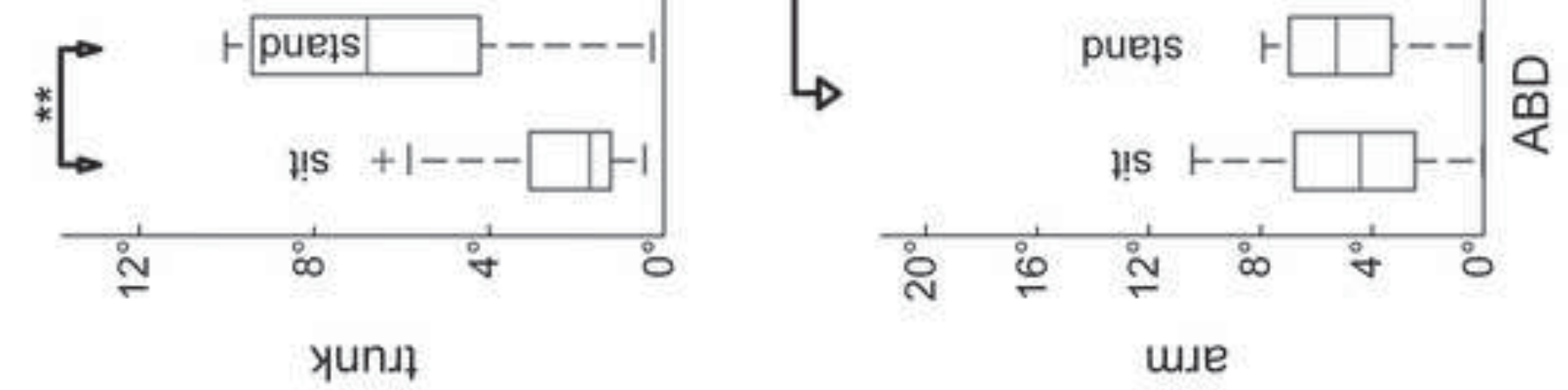
8. Supplementary Material

Click here to download 8. Supplementary Material: supplementary material.docx 
- A marker-less motion capture system was compared to a standard marker-based system.

- Functional upper extremity movements were assessed.

- Marker-less system with sufficient accuracy for clinical setting.

- Functional movements should be performed in sitting. 